\title{
Practice patterns for lower respiratory tract infections in hospital patients with particular focus on bacteriological examinations and injection antibiotics use
}

\author{
This article was published in the following Dove Press journal: \\ International Journal of General Medicine \\ 17 July 2013 \\ Number of times this article has been viewed
}

\section{Mikio Wadal \\ Takeo Nakayama ${ }^{2}$ \\ Tatsuro Ishizaki ${ }^{3}$ \\ Toshihiko Satoh ${ }^{4}$ \\ Shunya Ikeda ${ }^{5}$ \\ 'General Internal Medicine, Fukuchiyama City Hospital, Fukuchiyama, Kyoto, Japan; ${ }^{2}$ Department of Health Informatics, Kyoto University School of Public Health, Kyoto, Japan; \\ ${ }^{3}$ Tokyo Metropolitan Institute of Gerontology, Tokyo, Japan; ${ }^{4}$ School of Social Informatics, Aoyama Gakuin University, Sagamihara, Kanagawa, Japan; ${ }^{5}$ Department of Pharmaceutical Sciences, School of Pharmacy, International University of Health and Welfare, Ohtawara, Tochigi, Japan}

Correspondence: Mikio Wada

General Internal Medicine,

Fukuchiyama City Hospital,

23I Atsunakacho, Fukuchiyama,

Kyoto 620-8505, Japan

$\mathrm{Tel}+81773222101$

Fax +8I 77322618

Email mwada@d6.dion.ne.jp
Background: Various types of medical institutions perform treatments for lower respiratory tract infections. However, few studies have assessed and compared practice patterns across different medical institutions in Japan. To assess practice patterns for community-acquired lower respiratory tract infections among patients who needed hospitalization, we examined the use of injection antibiotics and the implementation of bacteriological examinations, and compared these across medical institutions. We then evaluated whether bacteriological examinations were being adequately implemented.

Methods: A cross-sectional study was conducted using a database of health insurance claims. Subjects were patients ( $\geq 16$ years old) who received injection antibiotics to treat lower respiratory tract infections, and who were listed among 2004-2007 insurance claims data. For each type of antibiotic, we obtained the dose prescribed, the number of patients treated, and the frequency of bacteriological examinations.

Results: A total of 1649 patients were evaluated. The implementation rate of Gram stain and sputum culture was $14 \%$ at clinics ( $<20$ beds), approximately $30 \%$ at small hospitals (20-99 beds), and 40\%-45\% at medium-sized (100-499 beds) and large hospitals ( $\geq 500$ beds). As a whole, beta-lactams were most frequently used, accounting for $80 \%$ of all antibiotics used. Among beta-lactams, penicillins comprised $25 \%$ of the total amount of drugs used at hospitals with $\geq 300$ beds, but approximately $13 \%$ at clinics and small hospitals.

Conclusion: Practice patterns varied depending on the size of the medical institution. The implementation rate of bacteriological examinations was not high enough, especially at clinics and small hospitals.

Keywords: treatment pattern, medical institution size, variability, health insurance claims

\section{Introduction}

The emergence of antibiotic-resistant bacteria and the continual increase in antibiotic resistance present major problems in the treatment of infectious diseases. The appropriate use of antibiotics is essential, given that their improper use promotes antibiotic resistance. ${ }^{1-3}$ One of the major problems underlying antibiotic use is the lack of consideration for the responsible pathogen, resulting in inappropriate use of antibiotics.

In Japan, the "Guidelines for the Management of Community-Acquired Pneumonia in Adults" were published by the Japanese Respiratory Society (JRS) in $2000,{ }^{4}$ and they were updated in 2007. ${ }^{5}$ These guidelines recommended that physicians review the appropriateness of antibiotics before use, and indicated that antibiotics should be 
used effectively by first determining the specific pathogen involved through bacteriological examinations.

Although lower respiratory tract infections are treated at various types of medical institutions, few studies have compared the practice patterns for such infections across medical institutions in Japan. Accordingly, this study aimed to assess practice patterns for community-acquired lower respiratory tract infections among hospital patients with regard to bacteriological examinations and injection antibiotic use. We then evaluated whether bacteriological examinations were being adequately implemented.

\section{Materials and methods Design and database}

With the universal health care coverage system, most people in Japan are covered under some type of health insurance plan, such as national health insurance, a health insurance society, or a mutual aid association. ${ }^{6}$ Almost all medical institutions provide health care services covered by health insurance, ${ }^{7}$ and they issue health insurance claims (hereafter, "claims data") every month for each patient. Claims data are inspected at the time of medical billing to determine whether services were adequate based on health insurance criteria. Information provided in claims data include patient characteristics (eg, name, sex, date of birth), name of medical institution, name of disease, treatments performed (eg, laboratory examinations, name of drugs and quantity, surgery), and health care charges.

In this cross-sectional study, we used the health insurance claims database of the Japan Medical Center Co, Ltd (JMDC), (Tokyo, Japan). The JMDC collects claims data of approximately 530,000 insured individuals from multiple health insurance societies in Japan. Data were obtained from each health insurance society under special security conditions. Information such as disease names, pharmaceutical products used, and treatments performed are entered in a standardized format using special techniques. ${ }^{8}$ Although patient characteristics in the JMDC database can be used to evaluate health care services, the database does not include physician characteristics (eg, age, sex, specialty). The JMDC database has been used in several published studies. ${ }^{9-11}$

\section{Study subjects}

Subjects were hospital patients aged 16 years or older who were listed in insurance claims submitted between January 2004 and December 2007, and who received injection antibiotic therapy for a lower respiratory tract infection.
Lower respiratory tract infections were defined as diseases corresponding to J13, J14, J15, J16, J17, J18, J20 (with the exception of J20.3-20.7), J21.9, J22, J40, and J69 according to the World Health Organization's International Classification of Diseases, tenth revision. ${ }^{12}$

The names of diseases recorded on claims data include primary diseases targeted mostly for treatment (hereafter, "primary disease;" more than one primary disease is possible, and some cases will have no record) and all other diseases (hereafter, "secondary disease;" more than one disease is possible, and some cases will have no record). Subjects of the present study were patients for whom:

Criteria 1: the primary disease was recorded and included the target disease of this study. The antibiotic used is indicated for the primary disease;

Criteria 2: the primary disease was recorded but was neither the target disease of this study nor a disease for which the antibiotic used was indicated. The secondary disease included the target disease of this study and the disease was the only disease for which the antibiotic used was indicated;

Criteria 3: the primary disease was not recorded, and the secondary disease included the target disease of this study and the disease was the only disease for which the antibiotic used was indicated.

Injection antibiotics approved in Japan, except for spectinomycin and sulfadimethoxine, were indicated for pneumonia and/or other lower respiratory tract infections, and they were assessed. The antibiotics assessed in this study are listed in the Supplementary material.

Data from the same individual in which the treatment period or medical institution performing the treatment differed were evaluated separately.

Exclusion criteria were: (1) patients for whom treatment extended beyond the target period; (2) nosocomial infections (ie, patients for whom treatment of the target disease began 4 days after hospitalization, or antibiotic administration was suspended for at least 3 days during the same hospitalization period [antibiotic treatment following suspension suggests a nosocomial infection]); and (3) long-term antibiotic therapy (eg, treatment for community-based pneumonia should be completed or drug modifications should be determined by the 14th day following treatment initiation. ${ }^{5}$ When certain bacteria [eg, intestinal bacteria] are considered responsible, 21 days of antibiotic administration may be recommended. ${ }^{13}$ We excluded patients who were treated with the same antibiotics for more than 21 days). 


\section{Evaluated items}

For each patient, we determined the type of antibiotic used, the dose and number of days the antibiotic was used, and whether bacteriological examinations were performed or not. Using the World Health Organization, Anatomical Therapeutic Chemical (ATC) classification as a reference, we classified antibiotics as penicillins, first-generation cephalosporins, second-generation cephalosporins, thirdgeneration cephalosporins, fourth-generation cephalosporins, carbapenems, aminoglycosides, macrolides, tetracyclines, lincomycins, fosfomycins, glycopeptides, quinolones, or other antibiotics. For drugs not registered with the ATC, we used classifications listed in the JRS guidelines. ${ }^{4}$ Because the appropriate dose varies considerably among drugs, normalized doses should be compared. The defined daily doses listed in the ATC are commonly used as standard doses; however, for some drugs, the standard dose listed in Japanese drug package inserts differs considerably from the defined daily doses of the ATC and, as mentioned above, some drugs are not registered with the ATC. Accordingly, in the present study, we defined the daily normal dose listed in Japanese drug package inserts as the standard unit (hereafter, "ddd"). When a range of normal doses was provided, we used the maximum daily limit of the normal dose. For example, the normal dose for ceftriaxone is given as $1-2 \mathrm{~g}$ /day, up to $4 \mathrm{~g}$ for severe patients; thus, we defined the standard dose as $2 \mathrm{~g}$ (ie, 1 ddd for ceftriaxone is $2 \mathrm{~g}$ ).

Bacteriological examinations analyzed were Gram stain, sputum culture, blood culture, bacterial sensitivity tests, and other tests that have the potential to identify the responsible bacteria (eg, tests that estimate the responsible bacteria from blood or urine, such as the streptococcal pneumonia urinary antigen test). We judged that a test was implemented if it was recorded in the claims data.

The precise number of beds for individual hospitals could not be determined due to JMDC privacy policies. Accordingly, we used hospital bed classifications according to the "Patient's Behavior Survey" of the Ministry of Health, Labor, and Welfare ${ }^{14}$ as a reference and grouped hospitals as follows: <20, 20-99, 100-199, 200-299, $300-499$, and $\geq 500$ beds. Data were provided based on this classification. Institutions with $<20$ beds were classified as clinics, 20-99 beds as small hospitals, 100-499 beds as medium-sized hospitals, and $\geq 500$ beds as large hospitals.

Because disease names recorded in claims data did not always agree with the actual clinical diseases diagnosed, additional analyses were conducted. In addition to the target patients who met the eligibility criteria for this study, we considered patients who would be possibly using antibiotics to treat lower respiratory tract infections. These patients, in addition to the target patients, constituted the patients in analysis 1 . We evaluated the implementation of bacteriological examinations and antibiotic use for the patients in analysis 1 .

The patients in analysis 1 included target patients and those whose disease in claims data satisfied the following: the target disease of this study was recorded as the primary disease or the secondary disease; if the primary or secondary disease included diseases other than the target disease of this study and the antibiotic used was indicated, those diseases were respiratory diseases only.

Logistic regression analysis was conducted to determine predictors of the implementation of bacteriological examinations. The odds ratio (OR) and $95 \%$ confidence interval (CI) were calculated, setting the implementation of Gram stain or sputum culture as the dependent variable (implement $=1$; not implement $=0$ ). Physician characteristics (eg, age, sex, specialty) were not analyzed since this information was not recorded in the database. Patient's sex, age, and the institution size were set as explanatory variables.

Statistical analysis was performed using STATA software, version 10.0 (StataCorp LP, College Station, TX, USA).

\section{Results}

A total of 1649 inpatients (criteria 1: $42.1 \%$, criteria 2: 30.7\% criteria $3: 27.2 \%$ ) were analyzed. Patient characteristics (age and sex) are shown in Table 1. The numbers of target patients based on medical institution size are shown in Table 2.

The implementation of bacteriological examinations is shown in Table 3. Doses (aggregated as ddd) for the different types of antibiotics are shown in Figure 1. The proportion of patients who received the respective drugs is shown on the graph. The implementation rate for Gram stain and

Table I Patient characteristics

\begin{tabular}{lll}
\hline Age (years) & Patients $(\mathbf{n})$ & \\
\cline { 2 - 3 } & Male & Female \\
\hline $16-25$ & 122 & 91 \\
$26-35$ & 190 & 135 \\
$36-45$ & 166 & 109 \\
$46-55$ & 137 & 88 \\
$56-65$ & 169 & 101 \\
$\geq 66$ & 123 & 218 \\
Total & 907 & 742
\end{tabular}

Abbreviation: $\mathrm{n}$, number. 
Table 2 Number of target patients and their proportion based on medical institution size

\begin{tabular}{lll}
\hline $\begin{array}{l}\text { Institution size } \\
\text { (number of beds) }\end{array}$ & Patients (number) & (\%) \\
\hline$<20$ & 91 & 5.5 \\
$20-99$ & 199 & 12.1 \\
$100-199$ & 289 & 17.5 \\
$200-299$ & 197 & 11.9 \\
$300-499$ & 518 & 31.4 \\
$\geq 500$ & 355 & 21.5 \\
Total & 1649 & 100 \\
\hline
\end{tabular}

sputum culture was $40 \%$. Particularly among patients who were treated by fourth-generation cephalosporins and/or carbapenems, the implementation rate for Gram stain and sputum culture was $50 \%$ and $52 \%$, respectively. Beta-lactams comprised $80 \%$ of the total amount of drugs used, and the proportion of patients who received beta-lactams was $83 \%$.

Treatment patterns by medical institution size were compared. The implementation of bacteriological examinations is shown in Table 4. The implementation rate of Gram stain and sputum culture was $14 \%$ at clinics, approximately $30 \%$ at small hospitals, and $40 \%-45 \%$ at medium-sized and large hospitals. Between-drug comparisons of doses are shown in Figure 2. The dose of each drug is shown as a proportion of the total amount of drug used as assessed by ddd (set to 100). Beta-lactams were most frequently used, comprising $80 \%$ of the total amount of drugs used. Among beta-lactams, penicillins comprised $25 \%$ of the total amount of drugs used at hospitals with $\geq 300$ beds, but $12 \%$ and $13 \%$ at clinics and small hospitals, respectively.

Analysis 1 targeted 1751 patients. The implementation rate for Gram stain and sputum culture in analysis 1 was $40 \%$. Those rates at clinics were $13 \%$ in analysis 1 . They were approximately $30 \%$ at small hospitals, and 40\%-45\% at medium-sized and large hospitals. Beta-lactams were most frequently used among patients for analysis 1, comprising $80 \%$ of the total amount of drugs used. In analysis 1 , penicillins comprised $23 \%$ of the total amount of drugs used at

Table 3 Implementation of bacteriological examinations (proportion of patients)

\begin{tabular}{ll}
\hline Patients $(\mathbf{n}=\mathbf{~ 1 6 4 9 )}$ & $\%$ \\
\hline Gram stain & 39.8 \\
Sputum culture & 39.8 \\
Blood culture & 23.4 \\
Sensitivity test & 18.1 \\
Other examinations & 24.7 \\
\hline
\end{tabular}

Abbreviation: $n$, number. hospitals with $\geq 300$ beds, and $11 \%$ and $12 \%$ at clinics and small hospitals, respectively.

As for the predictors of bacteriological examination implementation, ORs were 0.68 (95\% CI: 0.55-0.84) for females, 1.01 (95\% CI: 1.00-1.01) for increasing age, and 1.20 (95\% CI: 1.12-1.28) for large-sized institutions. Similar results were obtained when the implementation of sputum culture was used as a dependent variable.

\section{Discussion}

The present study with large claim database revealed that practice patterns for lower respiratory tract infections differed by medical institution size, and that implementation rates of bacteriological examinations were very low, especially at clinics and small hospitals in Japan.

In the present study, more than one-fifth of analyzed patients were treated with broad-spectrum antibiotics such as fourth-generation cephalosporins and/or carbapenems. Physicians sometimes have no choice but to prescribe these antibiotics for severe cases or under particular circumstances, even if they refer to JRS guidelines. The frequency of inappropriate antibiotics use should be assessed, although such analysis is beyond the scope of this study.

Previous studies in other countries have investigated the variability of antibiotic prescribed by physicians, reporting practice setting, practice volume, regional differences, physician age, and physician specialty as predictors of prescribing decisions. ${ }^{15-17}$ Differences in treatment by medical institution size were observed in the present study as well.

Lower respiratory tract infections are caused by bacteria as well as by viruses. One possibility is that patients treated in clinics and small hospitals had a higher likelihood of having viral infections or mild cases. However, in the present study, all analyzed patients were hospitalized and given antibiotics - these were the inclusion criteria of this study. Antibiotics are used to treat bacterial infections. When physicians use antibiotics, they should make efforts to estimate and/or identify responsible pathogens regardless of disease severity, and then use appropriate drugs.

The implementation rate of bacteriological examinations was generally low at clinics and small hospitals and higher at large hospitals. Nonetheless, less than half of the targeted patients underwent bacteriological tests. Surprisingly, even in the cases that broad-spectrum antibiotics such as fourthgeneration cephalosporins and/or carbapenems were used, the implementation rate for Gram stain and sputum culture was around $50 \%$ at most. This suggests that the practice of administering injection antibiotics without performing 


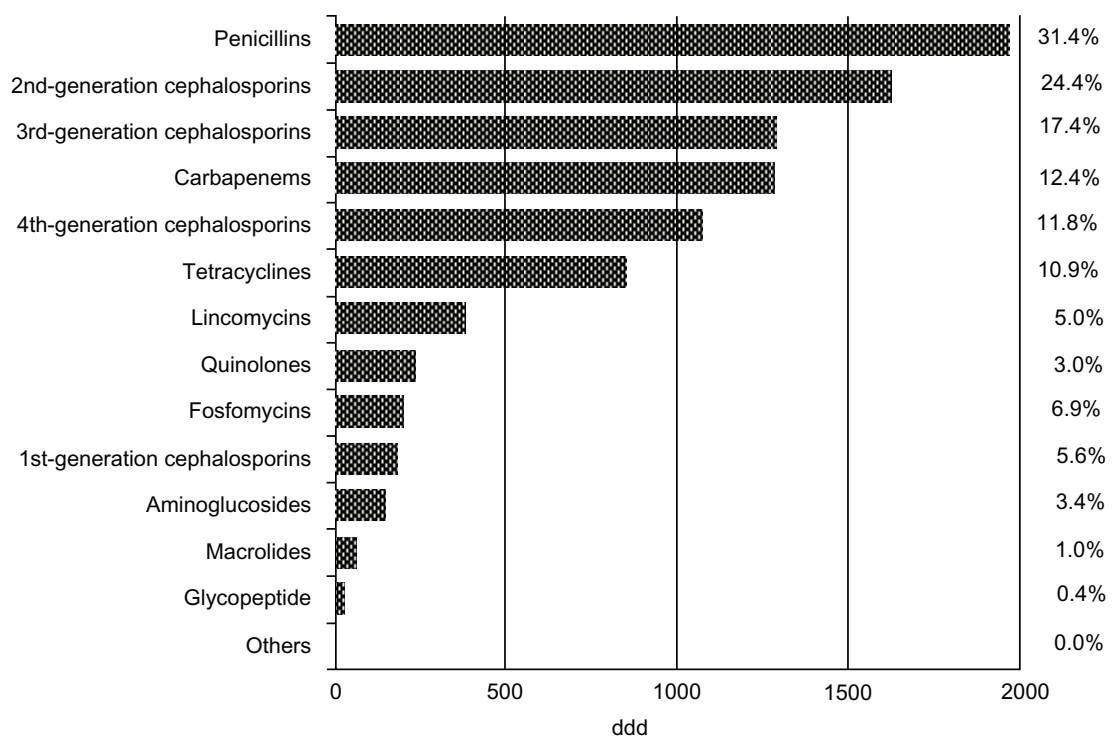

Figure I Normalized ddd of different antibiotics.

Notes: The right side of the graph shows the proportion of patients who used the antibiotic. The total percentage of patients exceeds $100 \%$ because injections of different drugs administered to the same patient were counted separately.

Abbreviation: ddd, defined daily dose.

Table 4 Implementation of bacteriological examinations based on medical institution size

\begin{tabular}{|c|c|c|c|c|c|c|}
\hline & \multicolumn{6}{|l|}{ Bed } \\
\hline & $1-19$ & $20-99$ & $100-199$ & $200-299$ & $300-499$ & $\geq \mathbf{5 0 0}$ \\
\hline \multicolumn{7}{|l|}{ Patient (\%) } \\
\hline Gram stain & 14.0 & 28.6 & 40.2 & 44.5 & 44.6 & 43.9 \\
\hline Sputum culture & 14.0 & 31.7 & 40.9 & 41.5 & 44.0 & 43.0 \\
\hline Blood culture & 4.3 & 13.6 & 14.8 & 27.5 & 23.9 & 38.5 \\
\hline Sensitivity test & 8.6 & 17.1 & 26.1 & 15.5 & 15.1 & 20.7 \\
\hline Other examinations & 11.8 & 13.1 & 22.7 & 22.0 & 28.9 & 31.6 \\
\hline
\end{tabular}

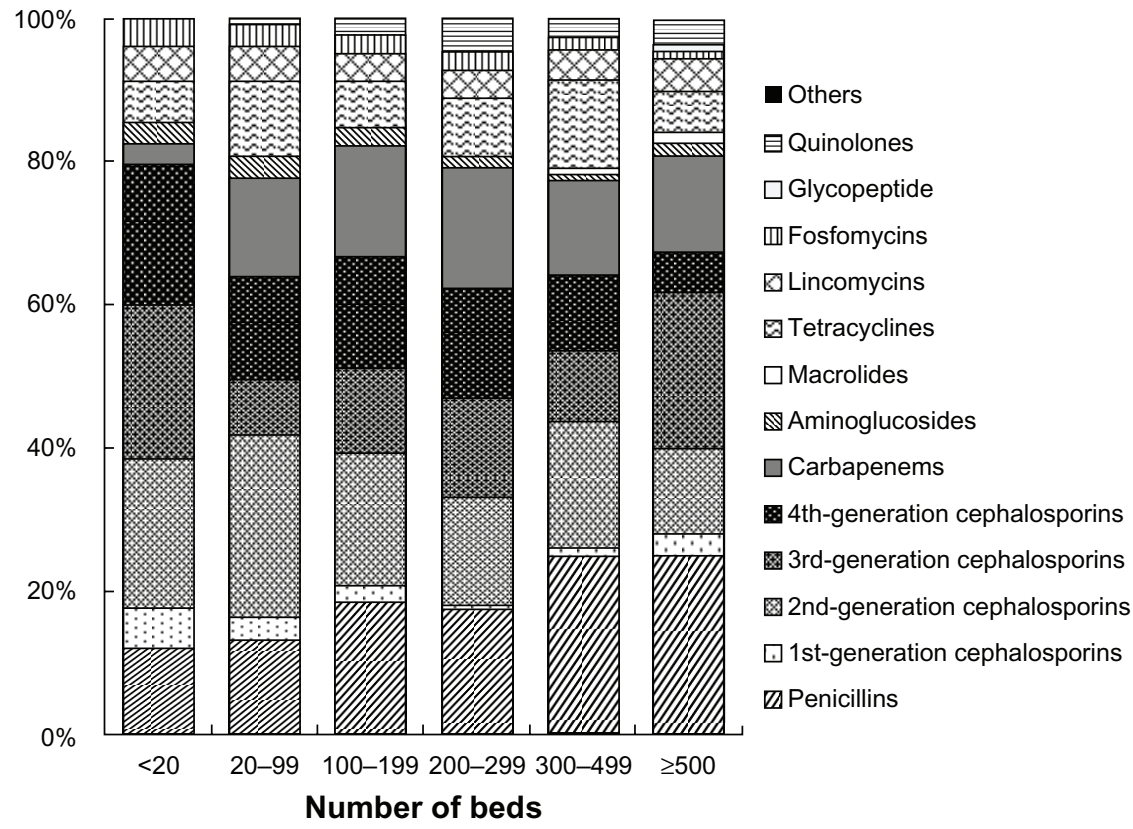

Figure 2 Between-drug comparisons of drug dose based on medical institution size. 
bacteriological examinations is widespread. Although logistic regression analysis revealed that men and older individuals were more likely to undergo Gram stain and sputum culture tests, the effect size was too small to be conclusive. Implementation of bacteriological examinations may depend on several factors, such as the physician's views regarding infectious diseases and the presence of a laboratory that can perform examinations. However, the analysis of these factors is beyond the scope of this study due to the limitation of the database used.

Some large hospitals in Japan reported that restricting antibiotic use and establishing a notification system successfully modified the use of broad-spectrum antibiotics. ${ }^{18-20} \mathrm{On}$ the other hand, restricting the use of specific antibiotics may increase strains resistant to other antibiotics. ${ }^{21}$ Merely establishing restricting and/or notification systems is insufficient, and may be difficult for some small institutions. In the practice of infectious diseases, individualized treatment is important. Gram stain is useful in the treatment of community-acquired pneumonia; $; 2$ yet, such tests are not performed to a sufficient degree. Physicians should perform bacteriological examinations to estimate and/or identify responsible pathogens, consider drug sensitivity, and prescribe the most appropriate antibiotics. De-escalation strategy, that is the practice as above mentioned, is one of the key strategies in antibiotic treatment. ${ }^{23-25}$ The JRS guidelines recommend against the use of broad-spectrum antibiotics by physicians without sufficient review of the medication's appropriateness, and also suggest that estimating and/or identifying the responsible pathogen would lead to the effective use of antibiotics. However, our findings suggest that many physicians failed to adhere to these recommendations. In addition to widely disseminating the guidelines, further efforts should be made to improve education on infectious diseases among physicians with various levels of experience. ${ }^{26}$ Given that medical service fees influence physicians' attitudes, ${ }^{27}$ one option might be to address medical profit.

There are some limitations to this study. First, the claims data used in this study were insurance claims rather than clinical records. Claims data are aggregated monthly; therefore, if a bacteriological examination and antibiotic administration occur in the same month, their order cannot be determined. Consequently, whether the bacteriological examination was conducted before or after antibiotic administration could not be differentiated, suggesting that bacteriological examinations before antibiotic treatment may be less frequent than the results of this study.
Second, the disease listed in claims data does not always match the clinical diagnosis. Claims data are inspected at the time of medical billing, and it is expected that the name of the disease for which the antibiotic is indicated will be recorded. Therefore, we conducted additional analyses based on the names of diseases provided in claims data. The implementation rate of bacteriological examinations and prescription patterns of injection antibiotics were similar between patients for analysis 1 and the target patients of this study. These results suggested that the present findings may accurately reflect clinical reality.

Third, the JMDC database used in this study consists of individuals covered by health insurance societies. Compared with the nation as a whole, this population is younger on average, suggesting that the present findings cannot be generalized to the entire country.

Fourth, severity and comorbidity in individual patients (ie, differences in case-mix) could not be assessed due to the limitation of the database. It is possible that patient characteristics at clinics and small hospitals were of a less severe case-mix compared to medium and large hospitals, and that patients were less likely to undergo bacteriological examination. Nonetheless, the implementation rates of Gram stain and sputum culture at clinics and small hospitals were critically low ( $14 \%$ to $30 \%$ ) for patients who required hospitalization. From the perspective of appropriate antibiotic use, physicians should make conscious efforts to estimate and/or identify responsible pathogens.

It was not until recently that claim databases became available for studies and that practice patterns could be analyzed, as was conducted in this study. Based on the present findings, we conclude that practice patterns varied by medical institution size. Moreover, bacteriological examinations were insufficiently implemented, especially at clinics and small hospitals. To improve the practice of treating lower respiratory tract infections, issues such as more thorough dissemination of current guidelines, increased education of infectious disease among physicians with various levels of experience, further evaluation of appropriate antibiotic use, effective interventions, and comparison of antibiotic resistance and compliance with guidelines should be addressed in the future.

\section{Acknowledgments}

We thank the Japan Medical Data Center Co, Ltd. (Shinya Kimura, CEO) for their extensive assistance and cooperation. This study is partly supported by the grant-in-aid by 
the Ministry of Education, Culture, Sports, Science and Technology in Japan.

\section{Disclosure}

The authors report no conflicts of interest in this work.

\section{References}

1. Fritsche TR, Sader HS, Toleman MA, Walsh TR, Jones RN. Emerging metallo-beta-lactamase-mediated resistances: a summary report from the worldwide SENTRY antimicrobial surveillance program. Clin Infect Dis. 2005;41 Suppl 4:S276-S278.

2. Goossens H, Ferech M, Vander Stichele R, Elseviers M; for ESAC Project Group. Outpatient antibiotic use in Europe and association with resistance: a cross-national database study. Lancet. 2005; 365(9459):579-587.

3. Suzuki S, Yamane K, Wachino J, Matsui M, Konda T, Arakawa Y. Three months survey of multidrug-resistant Enterobacteriaceae in Japan. Nihon Rinsho. 2012;70(2):187-191. Japanese.

4. The Japanese Respiratory Society Guidelines for the Management of Community-Acquired Pneumonia in Adults. Tokyo: The Japanese Respiratory Society; 2000. Japanese.

5. The JRS Guidelines for the Management of Community-Acquired Pneumonia in Adults. Tokyo: The Japanese Respiratory Society; 2007. Japanese.

6. Ikegami N, Yoo BK, Hashimoto H, et al. Japanese universal health coverage: evolution, achievements, and challenges. Lancet. 2011; 378(9796):1106-1115.

7. Hashimoto H, Ikegami N, Shibuya K, et al. Cost containment and quality of care in Japan: is there a trade-off? Lancet. 2011;378(9797): 1174-1182.

8. Kimura S, Sato T, Ikeda S, Noda M, Nakayama T. Development of a database of health insurance claims: standardization of disease classifications and anonymous record linkage. J Epidemiol. 2010; 20(5):413-419.

9. Kirigaya D, Nakayama T, Ishizaki T, Ikeda S, Satoh T. Management and treatment of osteoporosis in patients receiving long-term glucocorticoid treatment: current status of adherence to clinical guidelines and related factors. Intern Med. 2011;50(22):2793-2800.

10. Nakaoka S, Ishizaki T, Urushihara H, et al. Echocardiography for the detection of valvulopathy associated with the use of ergot-derived dopamine agonists in patients with Parkinson's disease. Intern Med. 2011;50(7):687-694.

11. Tanihara S, Okamoto E, Une H. Estimating medical expenditures spent on rule-out diagnoses in Japan. J Eval Clin Pract. 2012;18(2):426-432.

12. World Health Organization. [webpage on the Internet]. International Classification of Diseases (ICD). Available from: http://www.who.int/ classifications/icd/en/. Accessed May 20, 2013.
13. Gilbert DN, Moellering RC Jr, Eliopoulos GM, Henry F. Chambers HF, Saag MS, eds. The Sanford Guide to Antimicrobial Therapy. 41st ed. Virginia: Antimicrobial Therapy, Inc;2011.

14. Ministry of Health, Labour and Welfare. Patient's Behaviour Survey 2011. Available from:http://www.mhlw.go.jp/toukei/saikin/hw/ jyuryo/11/dl/kakutei-tyousa-gaiyo.pdf. Accessed May 20, 2013. Japanese.

15. Huang N, Chou YJ, Chang HJ, Ho M, Morlock L. Antibiotic prescribing by ambulatory care physicians for adults with nasopharyngitis, URIs, and acute bronchitis in Taiwan: a multi-level modeling approach. Fam Pract. 2005;22(2):160-167.

16. Steinman MA, Landefeld CS, Gonzales R. Predictors of broad-spectrum antibiotic prescribing for acute respiratory tract infections in adult primary care. JAMA. 2003;289(6):719-725.

17. Linder JA, Stafford RS. Antibiotic treatment of adults with sore throat by community primary care physicians: a national survey, 1989-1999. JAMA. 2001;286(10):1181-1186.

18. Ooe T, Abe Y. Effect of the notification system for the use of antimicrobial agents. Journal of Japanese Society of Hospital Pharmacists. 2007;43(8):1113-1116. Japanese.

19. Sakai Y, Inoue M, Arima C, Kubo H, Tsuruta M. Effect of introduction for specific antimicrobial reporting system. Kankyou Kansen. 2008;23(1):66-71. Japanese.

20. Ono Y, Ueda K, Shibatani T, Toukunaga Y, Shimizu J, Higashi T. Restriction program of carbapenems aimed at correct antimicrobial use. Kankyou Kansen. 12, 2007;22(4):286-293. Japanese.

21. Rahal JJ, Urban C, Horn D, et al. Class restriction of cephalosporin use to control total cephalosporin resistance in nosocomial Klebsiella. JAMA. 1998;280(14):1233-1237.

22. Sato T, Aoshima M, Ohmagari N, Tada H, Chohnabayashi N. Usefulness of sputum Gram staining in community-acquired pneumonia. Nihon Kokyuki Gakkai Zasshi. 2002;40(7):558-563. Japanese.

23. Höffken G, Niederman MS. Nosocomial pneumonia: the importance of a de-escalating strategy for antibiotic treatment of pneumonia in the ICU. Chest. 2002;122(6):2183-2196.

24. van der Eerden MM, Vlaspolder F, de Graaff CS, et al. Comparison between pathogen directed antibiotic treatment and empirical broad spectrum antibiotic treatment in patients with community acquired pneumonia: a prospective randomised study. Thorax. 2005;60(8): 672-678.

25. Alvarez-Lerma F, Alvarez B, Luque P, et al. Empiric broad-spectrum antibiotic therapy of nosocomial pneumonia in the intensive care unit: a prospective observational study. Crit Care. 2006;10(3):R78.

26. Ohmagari N. Antimicrobial stewardship. Nihon Rinsho. 2012; 70(2):298-304. Japanese.

27. Eisenberg JM, Williams SV. Cost containment and changing physicians' practice behavior. Can the fox learn to guard the chicken coop? JAMA. 1981;246(19):2195-2201. 


\section{Supplementary table}

Table SI Antibiotics list

\begin{tabular}{|c|c|}
\hline \multicolumn{2}{|l|}{ Classification } \\
\hline Penicillins & $\begin{array}{l}\text { benzylpenicillin, ampicillin/cloxacillin, ampicillin, aspoxicillin, piperacillin, sulbactam/ampicillin, tazobactam/ } \\
\text { piperacillin }\end{array}$ \\
\hline First-generation cephalosporins & cefalotin, cefazolin \\
\hline Second-generation cephalosporins & cefbuperazone, cefmetazole, cefminox, cefotetan, cefotiam, flomoxef \\
\hline Third-generation cephalosporins & $\begin{array}{l}\text { cefmenoxime, cefodizime, cefoperazone, cefotaxime, cefpiramide, cefsulodin, ceftazidime, ceftizoxime, } \\
\text { ceftriaxone, latamoxef, sulbactam/cefoperazone }\end{array}$ \\
\hline Fourth-generation cephalosporins & cefepime, cefozopran, cefpirome \\
\hline Carbapenems & biapenem, doripenem, imipenem/cilastatin, meropenem, panipenem/betamipron \\
\hline Aminoglycosides & $\begin{array}{l}\text { amikacin, arbekacin, astromicin, bekanamycin, dibekacin, gentamicin, isepamicin, kanamycin, micronomicin, } \\
\text { netilmicin, ribostamycin, sisomicin, tobramycin }\end{array}$ \\
\hline Macrolides & erythromycin, kitasamycin \\
\hline Tetracyclines & minocycline \\
\hline Lincomycins & clindamycin, lincomycin \\
\hline Fosfomycins & fosfomycin \\
\hline Glycopeptides & teicoplanin, vancomycin \\
\hline Quinolones & ciprofloxacin, pazufloxacin \\
\hline Other antibiotics & aztreonam, carmonam, chloramphenicol, linezolid, sulfadiazine/trimethoprim \\
\hline
\end{tabular}

\section{Publish your work in this journal}

The International Journal of General Medicine is an international, peer-reviewed open-access journal that focuses on general and internal medicine, pathogenesis, epidemiology, diagnosis, monitoring and treatment protocols. The journal is characterized by the rapid reporting of reviews, original research and clinical studies across all disease areas.
A key focus is the elucidation of disease processes and management protocols resulting in improved outcomes for the patient.The manuscript management system is completely online and includes a very quick and fair peer-review system. Visit http://www.dovepress.com/ testimonials.php to read real quotes from published authors. 